Gut, 1987, 28, 323-329

\title{
Comparative effectiveness of the tumour diagnostics, CA 19-9, CA 125 and carcinoembryonic antigen in patients with diseases of the digestive system
}

\author{
K SAKAMOTO, Y HAGA, R YOSHIMURA, H EGAMI, Y YOKOYAMA, \\ AND M AKAGI \\ From the Department of Surgery II, Kumamoto University Medical School, Kumamoto, Japan
}

SUMmARY Serum concentrations of CA 19-9, CA 125 and carcinoembryonic antigen (CEA) in 145 patients with gastrointestinal carcinomas and 89 with non-neoplastic diseases were determined to compare the clinical usefulness of these tumour markers. Significantly fewer positive cases were obtained with serum CA $19-9(9 \%)$ and CA $125(8 \%)$ tests than the CEA test $(22 \%)($ both p $<0.05)$ in patients with benign diseases, while comparable sensitivities were achieved with the CA 19-9 $(44 \%)$ test, the CA $125(41 \%)$ test and the CEA test $(47 \%)$ in those with a carcinoma. High incidences of raised concentrations of serum CA 19-9 and CA 125 were observed in case of cancer of the pancreas (CA 19-9: 87\%, CA 125: 67\%) and biliary tract (CA 19-9: 63\%, CA 125: 48\%). Combined tests of CA $19-9$ and CA 125 revealed increments in the sensitivity $(61 \%)$ and provided a higher specificity $(87 \%)$ than that of the single CEA test $(78 \%)$. These combined tests were most useful for a differential diagnosis of pancreatic carcinoma ( $97 \%$ positive) and biliary tract carcinoma $(74 \%)$ from chronic pancreatitis $(4 \%)$ and cholelithiasis $(0 \%)$, respectively. Studies on the relations of clinical staging and serum concentrations of CA 19-9 and CA 125 revealed significant rises in cases of disseminated carcinoma. These results clearly show that serum CA 19-9 and CA 125 tests are most pertinent for diagnosing advanced carcinomas of organs in the digestive system.

Conventional tumour markers such as CEA and alpha-fetoprotein are used to monitor treatment and detect recurrence ${ }^{1-\phi}$ of malignancy. These tumour markers also show a non-specific reactivity in benign diseases $^{\text {s. }}$ and sometimes in even normal individuals. $^{6}{ }^{\mathrm{B}}$

Attempts have been made to search for monoclonal antibodies which might be used as tumour markers. CA 19-9, an antigenic determinant defined by murine monoclonal antibody $1116 \mathrm{NS} 19-9$, was generated by somatic hybridisation of the mouse myeloma cell line and splenocytes from a mouse

Address for correspondence: Kiyoshi Sakamoto, MD. Department of Surgery II, Kumamoto University Medical School, Kumamoto 860, Japan.

Received for publication 20 Junc 1986. immunised with human colorectal carcinoma cell line, SW1116. ${ }^{\circ}$ CA 125 is an antigenic determinant defined by murine monoclonal antibody $\mathrm{OC} 125$ developed by hybridisation techniques after immunisation of human epithelial ovarian carcinoma cell line OVCA433. "' These tumour markers have diagnostic value in cases of carcinomas of the digestive system, particularly of the pancreas. ${ }^{11-15}$

Clinical data on concomitantly measured serum concentrations of CEA, CA 19-9 and CA 125, including their sensitivity and specificity, have apparently not been documented.

We measured serum CA 19-9, CA 125 and CEA values in 145 patients with a malignancy in the digestive system and 89 with non-malignant diseases of the GI tract, the objective being to compare the clinical utility for diagnosis and treatment. 


\section{Methods}

\section{SUBJECTS}

Serum samples were obtained from patients in the Department of Surgery II, Kumamoto University Medical School and affiliated hospitals.

One hundred and forty five patients with histologically proven malignant disease and 89 with benign diseases of the digestive system were studied. The diagnoses of benign diseases were established by standard clinical, radiological and laboratory methods. The malignant diseases included carcinomas of the pancreas (30), biliary tract (23), liver (16), colon and rectum (19), stomach (45) and oesophagus (12). To investigate the relationship between the clinical staging and tumour markers, all patients with cancers of the pancreas, colon and rectum, and stomach were presumably divided into three groups as follows. Group 1: localised cancer without regional lymph node metastasis, group 2: localised cancer with regional lymph node metastasis, group 3: spread to liver, lung, peritoneum or bone.

Eighty nine patients with benign diseases included 27 with chronic pancreatitis, four with acute pancreatitis, 20 with chronic active hepatitis, 14 with liver cirrhosis, 11 with peptic ulcer and 13 with cholelithiasis. Chronic pancreatitis was diagnosed by

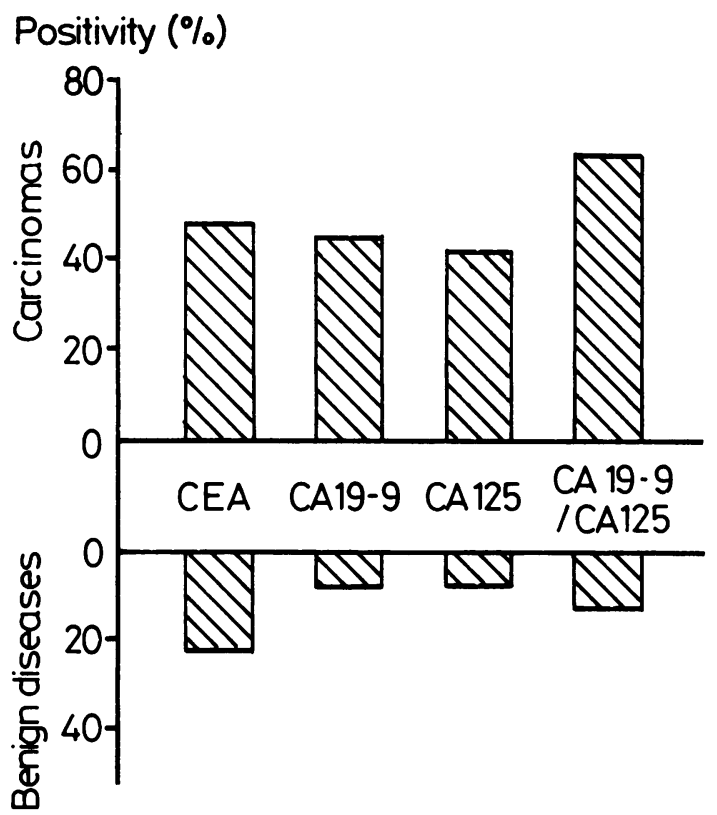

Fig. 1 Comparison of positivities of tumour markers $C A$ 19-9, CA 125 and CEA in 145 patients with digestive carcinomas and 89 patients with benign digestive diseases. Positivity of CEA is significantly higher than those of CA 19-9 and CA 125 in benign diseases. radiological signs of pancreatic calcification, exocrine dysfunction indicated by the pancreozymin-secretin test or by demonstration of moderate or high grade of endoscopic retrograde pancreatography (ERP) findings, by the grading of Braganza et al. . $^{16}$

Serum samples were stored at $-20^{\circ} \mathrm{C}$ and used within six months.

\section{ASSAY OF CA $19-9$}

CA 19-9 antigen concentration $(\mathrm{U} / \mathrm{ml})$ was measured by a forward sandwich radioimmunometric assay using a Centocor RIA kit (Centcor Inc, Malvern, PA, USA). A cutoff value of $37 \mathrm{U} / \mathrm{ml}$ was used. ${ }^{\text {? }}$

\section{ASSAY OF CA 125}

CA 125 antigen concentration $(\mathrm{U} / \mathrm{ml})$ was measured by a simultaneous sandwich solid phase radioimmunoassay of Centocor CA 125 RIA kit (Centocor Inc, Malvern, PA, USA). A cutoff value of $32 \mathrm{U} / \mathrm{ml}$ was used for women over 50 years of age and all men, and $143 \mathrm{U} / \mathrm{ml}$ was used for women under 49 , according to our previous investigation. ${ }^{17}$

\section{ASSAY OF CEA}

Carcinoembryonic antigen values were measured using the CIS I 125 RIA kit (CEA IRE SORIN, France) by the double antibody method. The upper limit of normal CEA was defined as $12.5 \mathrm{ng} / \mathrm{ml}$, as obtained by Booth et al. ${ }^{8}$

$\mathrm{p}$ Values were determined by Student's $t$ test.

\section{Results}

POSITIVITIES OF INDIVIDUAL TUMOUR MARKER TESTS

As shown in Table 1 and Fig. 1, among all patients with carcinomas, CEA, CA 19-9 and CA 125 tests showed comparable positivities $(47 \%, 44 \%$, and $41 \%$, respectively). In contrast, among those with non-neoplastic disease, the positivity of CEA (22\%) was significantly higher than that for CA $19-9(9 \%)$ and CA $125(8 \%)$ (both $p<0.05)$. The incidence of raised concentrations of serum CA 19-9 distributed among $6-87 \%$ of those with malignant disease and high incidences were observed both in pancreatic carcinoma $(26 / 30,87 \%)$ and biliary tract carcinoma $(14 / 23,61 \%)$. On the other hand, among those with a benign disease, the incidence ranged from $0-25 \%$. One of four patients with acute pancreatitis had a value of $290 \mathrm{U} / \mathrm{ml}$ which reverted to normal after treatment. Three $(21 \%)$ of 14 with liver cirrhosis had positive values. On the contrary, lower positivities were found in those with chronic pancreatitis (one of $27,4 \%$ ) and in two $(10 \%)$ of 20 patients with chronic active hepatitis. None of 13 with cholelithiasis was positive. Similarly, a high incidence of elevated 
Table 1 Positivities of individual tumour marker tests in patients with diseases of the digestive system

\begin{tabular}{|c|c|c|c|c|}
\hline \multirow[t]{2}{*}{ Diseases } & \multirow{2}{*}{$\begin{array}{l}\text { Patients } \\
\text { (n) }\end{array}$} & \multicolumn{3}{|c|}{ Positivities (\%) } \\
\hline & & $C A 19.9$ & $C A 125$ & $C E A$ \\
\hline \multicolumn{5}{|l|}{ Carcinomas } \\
\hline Pancreas & 30 & 87 & 63 & 67 \\
\hline Biliary tract & 23 & 61 & 48 & 39 \\
\hline Liver & 16 & 6 & 44 & 31 \\
\hline Colon/rectum & 19 & 47 & 32 & 68 \\
\hline Stomach & 45 & 27 & 36 & 38 \\
\hline Oesophagus & 12 & 17 & 0 & 33 \\
\hline \multicolumn{5}{|l|}{ Benign discases } \\
\hline Chronic pancreatitis & 27 & 4 & 0 & 26 \\
\hline Acute pancreatitis & 4 & 25 & 25 & () \\
\hline $\mathrm{CAH}$ & 20 & 10 & 10 & 10 \\
\hline Liver cirrhosis & 14 & 21 & 29 & 50 \\
\hline Peptic ulcer & 11 & 9 & 0 & 27 \\
\hline Cholelithiasis & 13 & 0 & 0 & 8 \\
\hline
\end{tabular}

CAH: chronic active hepatitis. Serum concentrations of the three markers were studied in the same sample.

values of serum CA 125 was observed both in cases of pancreatic carcinoma $(19 / 30,63 \%)$ and biliary tract carcinoma $(11 / 23,48 \%)$. In benign diseases, the incidence ranged from $0-29 \%$ and a high incidence of $29 \%$ (four of 14 ) was observed in liver cirrhosis. No patient with chronic pancreatitis, cholelithiasis or peptic ulcer had raised concentrations of serum CA 125. High incidences of raised values of CEA were seen in patients with colorectal cancer $(13 / 19,68 \%)$ and pancreatic cancer $(20 / 30,67 \%)$. Frequent positivities were observed in patients with liver cirrhosis (seven of $14,50 \%$ ), peptic ulcer (three of 11 , $27 \%$ ) and chronic pancreatitis (seven of $27,26 \%$ ).

\section{POSITIVITIES OF COMBINED TUMOUR MARKER} TESTS

Data obtained with three combinations of tests (CA 19-9/CEA, CA 125/CEA and CA 19-9/CA 125) were compared. As shown in Table 2, all with carcinoma showed similar sensitivities (CA 19-9/CEA: 65\%, CA 125/CEA: 64\%, CA 19-9/CA 125: 61\%). On the other hand, a higher specificity was found in CA 19-9/CA 125 tests $(87 \%)$ than those of CA 19-9/CEA test $(72 \%)$, CA $125 /$ CEA test $(72 \%)$ and even the single CEA test $(78 \%)$. Among patients with carcinoma, CA $19-9 /$ CA 125 proved positive in $97 \%$ of patients with pancreatic carcinoma, $74 \%$ of those with biliary tract carcinoma and $15-58 \%$ of other carcinomas. In benign diseases, only one $(4 \%)$ of 27 patients with chronic pancreatitis and no patient with cholelithiasis were positive in these combined tests, although one of four patients with acute pancreatitis and six $(43 \%)$ of 13 patients with liver cirrhosis showed raised concentrations of serum CA 19-9/CA 125.
CORRELATION OF SERUM CONCENTRATIONS OF CA $19-9$, CA 125 AND CEA

The correlation between CA 19-9 and CA 125 in 145 patients with malignant disease is shown in Figure 2. No significant correlation was observed between serum levels of CA $19-9$ and CA $125(r=0 \cdot 195)$. There was also no significant correlation between those of CA 19-9 and CEA $(r=0 \cdot 271)$ or between those of CA 125 and CEA $(r=0 \cdot 124)$.

\section{RELATIONSHIP OF SERUM LEVELS OF TUMOUR} MARKER AND TUMOUR STAGES IN PATIENTS WITH CARCINOMAS OF THE PANCREAS, STOMACH AND LARGE INTESTINE

The relation of tumour marker levels and tumour stages was studied in the three groups previously defined. As shown in Figure 3, values of serum CA 19-9 for group 3 were significantly higher than those for groups 1 and 2 in all patients with carcinomas of the pancreas, stomach, and large intestine $(p<0 \cdot 05)$. Values for groups 1 and 2, however, were raised as well as those for group 3 in case of pancreatic cancer. This was not observed with CA 125 or CEA. As shown in Figure 4, CEA values for advanced cancers (group 3) showed higher concentrations in comparison with localised carcinomas (groups 1 and 2), although no statistical significance was obtained. Relationship of serum CA 125 concentrations and tumour stages among the same patients was previously studied. ${ }^{15}$ It was concluded that in metastatic carcinomas, the CA 125 values were higher than in localised carcinomas.

Table 2 Positivities of combined tumour marker tests in patients with diseases of the digestive system

\begin{tabular}{|c|c|c|c|c|}
\hline \multirow[t]{2}{*}{ Diseases } & \multirow{2}{*}{$\begin{array}{l}\text { Patients } \\
(n)\end{array}$} & \multicolumn{3}{|c|}{ Positivities (\%) } \\
\hline & & $\begin{array}{l}C A 19-9 \\
/ C E A\end{array}$ & $\begin{array}{l}C A 125 \\
/ C E A\end{array}$ & $\begin{array}{l}C A 19-9 \\
/ C A 125\end{array}$ \\
\hline \multicolumn{5}{|l|}{ Carcinomas } \\
\hline Pancreas & 30 & 93 & 87 & 97 \\
\hline Biliary tract & 23 & 74 & 61 & 74 \\
\hline Liver & 16 & 38 & 63 & 44 \\
\hline Colon/rectum & 19 & 68 & 74 & 58 \\
\hline Stomach & 45 & 56 & 56 & 49 \\
\hline Oesophagus & 12 & 42 & 33 & 17 \\
\hline Total & 145 & 65 & 64 & 61 \\
\hline \multicolumn{5}{|l|}{ Benign diseases } \\
\hline Chronic pancreatitis & 27 & 30 & 26 & 4 \\
\hline Acute pancreatitis & 4 & 25 & 25 & 25 \\
\hline $\mathrm{CAH}$ & 20 & 15 & 20 & 15 \\
\hline Liver cirrhosis & 14 & 57 & 64 & 43 \\
\hline Peptic ulcer & 11 & 36 & 27 & 9 \\
\hline Cholelithiasis & 13 & 8 & 8 & 0 \\
\hline Total & 89 & 28 & 28 & 13 \\
\hline
\end{tabular}

$\mathrm{CAH}$ : chronic active hepatitis. Serum concentrations of the three markers were studied in the same sample. 


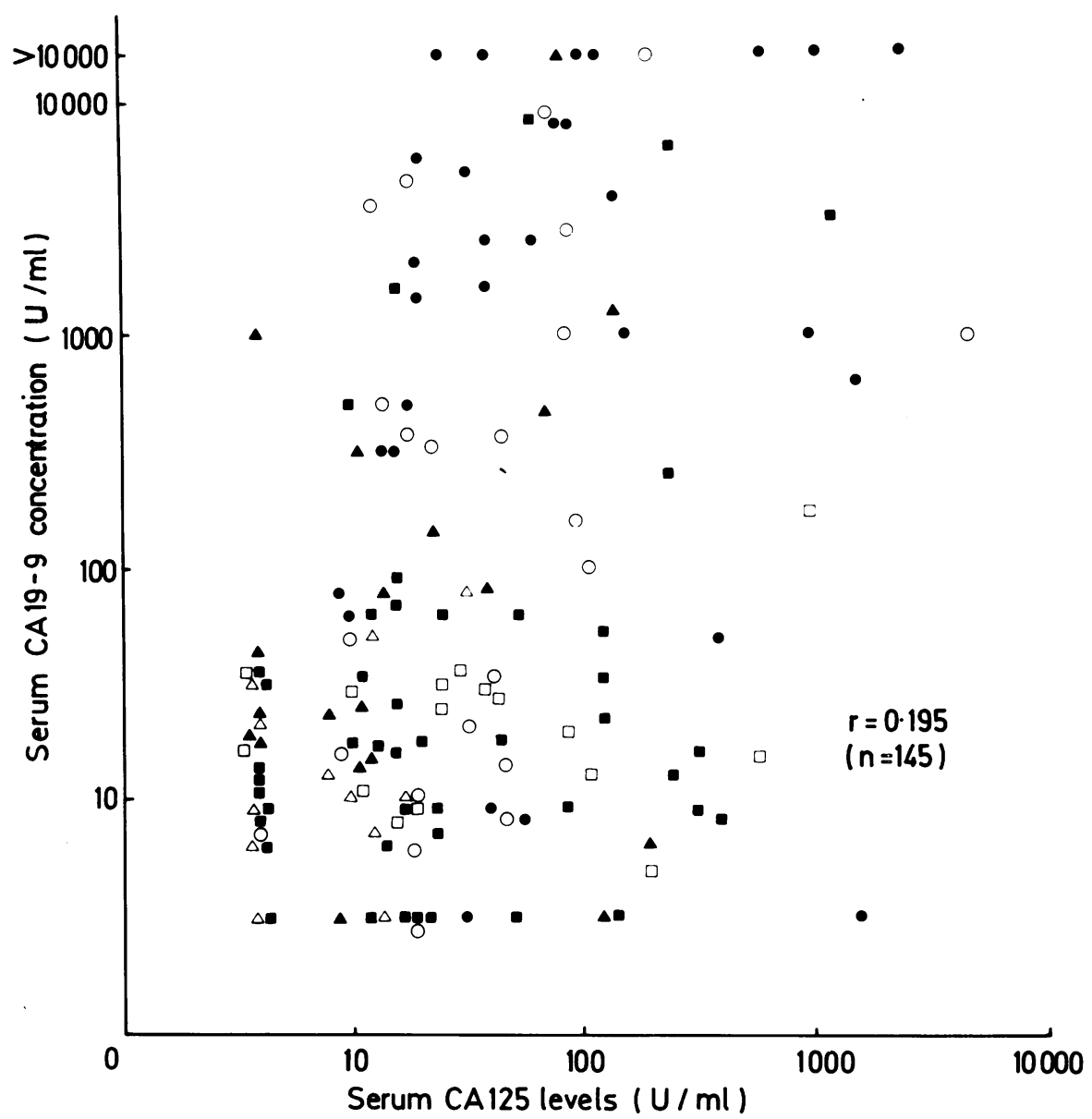

Fig. 2 Correlation between serum concentrations of CA 19-9 and CA 125 in patients with digestive carcinomas. $\mathbf{O}$ : pancreatic carcinoma; $\mathbf{\square}$ : stomach carcinoma; $\mathbf{\Delta}$ : colorectal carcinoma; $\bigcirc$ : carcinoma of the biliary tract; $\square$ : hepatocellular carcinoma; $\triangle$ : oesophageal . carcinoma.

\section{Discussion}

We measured serum values of the tumour markers CA $19-9$ and CA 125 in the same samples from patients with diseases of the digestive organs, in order to evaluate the clinical utility, compared with the conventional tumour marker, CEA. CA 19-9 and CA 125 tumour marker tests revealed similar positivities to CEA in all patients with malignant disease, although each marker had different positivity patterns (Table 1, Fig. 1). Interestingly, in oesophageal carcinoma, lower positivities were found with all the three tumour markers presumably because this lesion arises in squamous epithelium.

Importantly, CA 19-9 and CA 125 tests had significantly lower false positivities for non- neoplastic diseases of the digestive system compared with CEA (Table 1, Fig. 1). Major problems with the CEA test are high false positivities among patients with various benign diseases and even in apparently disease free heavy smokers. ${ }^{578}$

Positivities of serum CA 19-9 in patients with chronic pancreatitis vary in previous findings between 0 and $16 \% .^{1218}$ Our findings resemble those of Del Villano et al, ${ }^{12}$ but an increased positivity was reported by Schmiegel et al ${ }^{18}(14 \%)$. Schmiegel et al may have included patients with severe chronic pancreatitis such as the acute phase of chronic recurrent pancreatitis. We found transient rises of serum CA 19-9 concentrations in patients with acute pancreatitis, thereby suggesting that serial tests may be needed to exclude such conditions. A recent study 


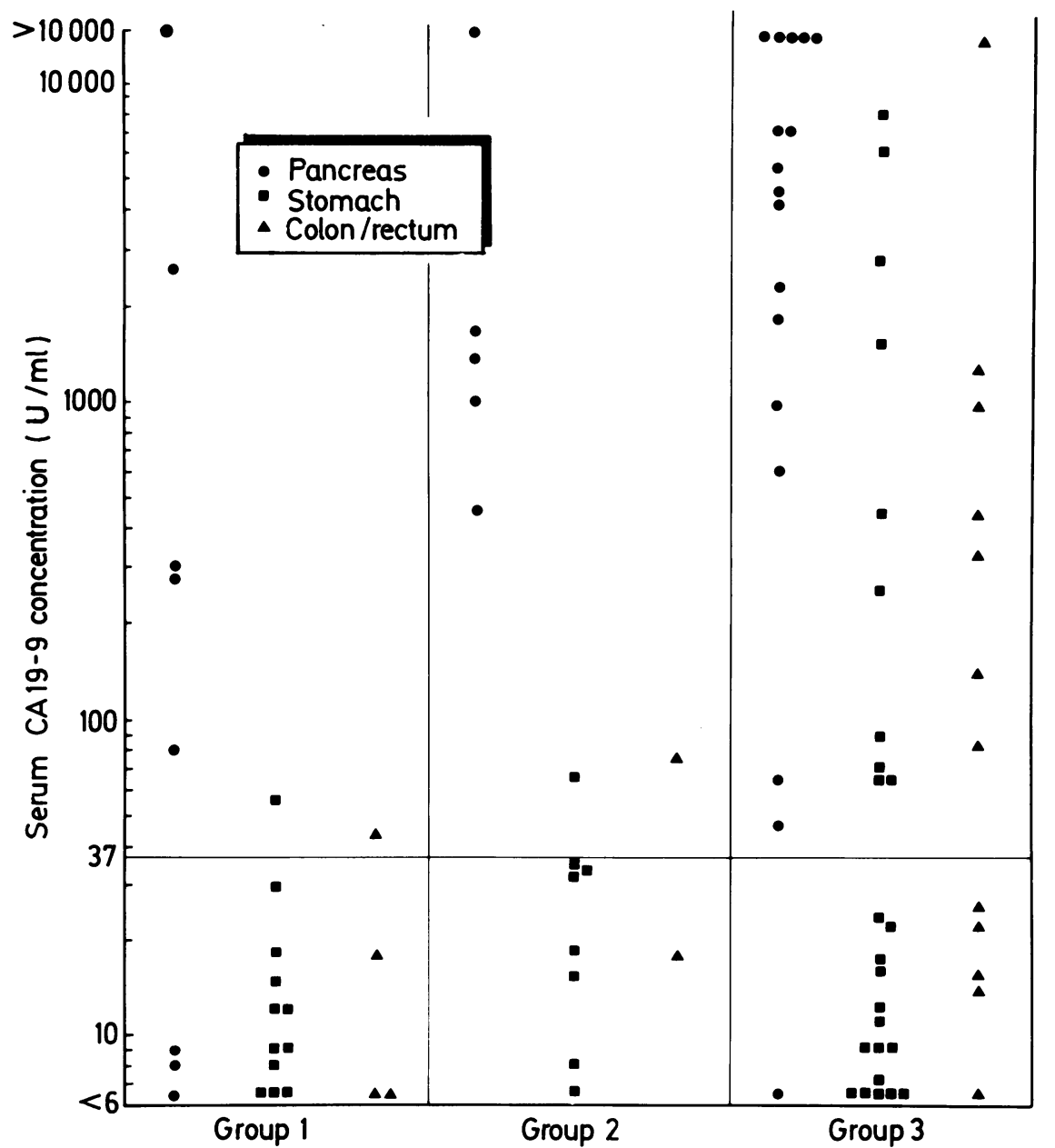

Fig. 3 Relationship of serum CA 19-9 and tumour stages in patients with gastrointestinal carcinomas. Group 1: localised cancer without regional lymph node metastasis. Group 2: localised cancer with regional lymph node metastasis. Group 3: spread to liver, lung, peritoneum or bone. Horizontal line represents the cutoff value of CA 19-9 in serum. : pancreatic carcinoma; $\mathbf{0}$ : stomach carcinoma; $\mathbf{\Delta}$ : colorectal carcinoma.

by Schmiegel et $a l^{18}$ revealed raised concentrations of CA 19-9 in pancreatic juice from normal controls and patients with pancreatitis as well as those with pancreatic cancer. This would suggest that transfer of CA 19-9 antigen from pancreatic juice into the circulation may cause a transient rise of serum CA 19-9 during the acute phase of pancreatitis. Similarly, we found a high concentration of CA 19-9 (7000$>10000)$ in bile as well as pancreatic juice from patients with cholelithiasis and carcinoma of the biliary tract and pancreas. As suggested by Jalanko et $a l,{ }^{19}$ stasis of bile with a high concentration of CA 19-9 may lead to a moderate rise of serum CA 19-9 in patients with obstructive jaundice. Furthermore, raised concentrations of CA 19-9, both in pancreatic juice and bile from normal controls and patients with benign diseases suggest that CA 19-9 in these juices may not have diagnostic value. Patients with benign liver diseases, especially liver cirrhosis, had high false positivities of CA 19-9, CA 125 and CEA, thereby indicating that those with benign liver diseases should be carefully followed.

Combined tests of multiple tumour markers may provide both higher sensitivities and lower specificities than a single test, hence we must consider the increment of false positive rate in benign disease 


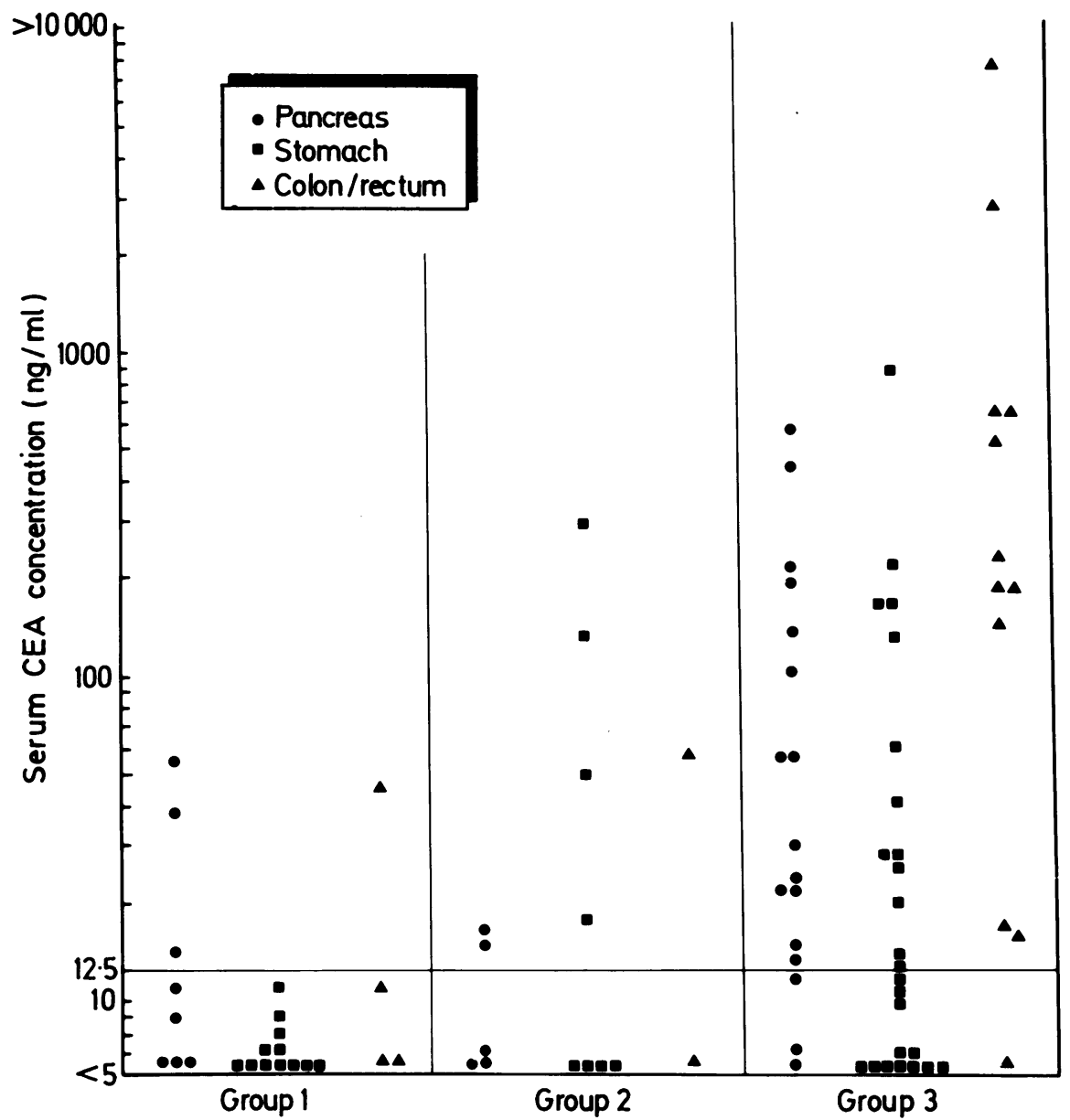

Fig. 4 Relationship of serum CEA concentrations and tumour stages in patients with gastrointestinal carcinomas. Group 1: localised cancer without regional lymph node metastasis. Group 2: localiséd cancer with regional lymph node metastasis. Group 3: spread to liver, lung, peritoneum or bone. Horizontal line represents the cut-off value of CEA in serum. CEA: carcinoembryonic antigen. 0 : pancreatic carcinoma; $\mathbf{a}$ : stomach carcinoma; $\mathbf{\Delta}$ : colorectal carcinoma.

when evaluating combined tests. Of importance was the finding that combined tests of CA 19-9 and CA 125 showed a lower false positivity (13\%) than did a single CEA test $(22 \%)$. Moreover, $97 \%$ of the patients with pancreatic cancer and $74 \%$ of those with cancer of the biliary tract were defined positive by the combination of CA 19-9 and CA 125. In contrast, no patient with cholelithiasis and only one patient with chronic pancreatitis was positive by the same combination of tests (Table 2). These results suggest that this combination will aid in differentiating carcinomas of the pancreas and of the biliary tract from chronic pancreatitis and cholelithiasis, respectively.
Tumour markers CA 19-9, CA 125, and CEA have proven to be useful in monitoring the effect of therapy and are pertinent prognostic tools for clinical use. ${ }^{1-5 \mid 10-1514-21}$ In the present study, the values for CEA in disseminated cancer were higher than in those with localised cancer. ${ }^{5}$ The same tendency was noted with CA 125 , in our previous report. ${ }^{\text {is }}$ The present work demonstrated the relation of CA 19-9 values and tumour stages among patients with carcinomas of the pancreas, stomach and large intestine. In cases of pancreatic carcinoma, high values of CA 19-9 were noted in localised as well as disseminated cancer. The pancreatic cancer patients in our study were advanced cases (except for two who 
underwent curative resection), as is usually the case when these tumors are diagnosed. Our previous and present studies revealed, however, that both CEA and CA 125 had less utility than CA 19-9 in the same patient with localised pancreatic carcinoma. These observations indicate that the CA 19-9 test is more sensitive than CEA or CA 125 for detection of localised cancer of the pancreas. Two patients with localised small carcinomas of the papilla of Vater showed respective serum CA 19-9 concentrations of 420 and $48 \mathrm{U} / \mathrm{ml}$ returning to normal $(<13 \mathrm{U} / \mathrm{ml})$ after pancreaticoduodenectomy (unpublished data). This result indicates that the CA 19-9 test may allow detection of resectable cancer. Among patients with carcinomas of the stomach and large intestine, CA 19-9 values were significantly higher in disseminated cancer than in cases of localised cancer. Similar data were reported by Kuusela et al, ${ }^{21}$ in patients with colorectal cancer. Therefore the CA 19-9 test may not be of use for detection of gastric or colorectal cancer in the early stage.

We thank M Ohara (Kyushu University) for comments on the manuscript.

\section{References}

1 Herrera MA, Chu TM, Holyoke ED. Carcinoembryonic antigen (CEA) as a prognostic and monitoring test in clinically complete resection of colorectal carcinoma. Ann Surg 1976; 183: 5-9.

2 Wanebo HJ, Rao B, Pinsky CM, et al. Preoperative carcinoembryonic antigen level as a prognostic indicator in colorectal cancer. N Engl J Med 1978; 299: 448-51.

3 Wanebo HJ, Stearns M, Schwartz MK. Use of CEA as an indicator of early recurrence and as a guide to a selected second-look procedure in patients with colorectal cancer. Ann Surg 1978; 188: 481-93.

4 Minton JP, Martin EW. The use of serial CEA determinations to predict recurrence of colon cancer and when to do a second-look operation. Cancer $1978 ; 42: 1422-7$.

5 Go VLW, Zamcheck N. The role of tumor markers in the management of colorectal cancer. Cancer 1982; 50: $2618-23$.

6 Ruoslahti E, Seppala M. Alpha-fetoprotein in cancer and fetal development. Adv Cancer Res 1979; 29: 275-345.

7 Laurence DJR, Stevens U, Bettelheim R, et al. Role of plasma carcinoembryonic antigen in diagnosis of gastrointestinal, mammary, and bronchial carcinoma. $\mathrm{Br}$ Med J 1972; 9: 605-9.
8 Booth SN, King JPG, Leonard JC, et al. Serum carcinoembryonic antigen in clinical disorders. Gut 1973; 14: 794-9.

9 Koprowski H, Steplewski Z, Mitchell K, et al. Colorectal carcinoma antigens detected by hybridoma antibodies. Somat Cell Genet 1979; 5: 957-72.

10 Bast RC, Feeney M, Lazarus $\mathrm{H}$, et al. Reactivity of a monoclonal antibody with human ovarian carcinoma. J Clin Invest 1981; 68: 1331-7.

11 Herlyn M, Sears HF, Steplewski Z, Koprowski H. Monoclonal antibody detection of a circulating tumorassociated antigen. I. Presence of antigen in sera of patients with colorectal, gastric, and pancreatic carcinoma. J Clin Immunol 1982; 2: 135-40.

12 Del Villano BC, Brennan S, Brock P, et al. Radioimmunometric assay for a monoclonal antibody-defined tumor marker, CA 19-9. Clin Chem 1983; 29: 549-52.

13 Bast RC, Jr, Klug TL, John E, et al. A radioimmunoassay using a monoclonal antibody to monitor the course of epithelial ovarian cancer. $N$ Engl J Med 1983; 309: 883-7.

14 Klug TC, Bast RC Jr, Niloff JM, Knapp RC, Zurawski VR Jr. Monoclonal antibody immunoradiometric assay for an antigenic determinant (CA 125) associated with human epithelial ovarian carcinomas. Cancer Res 1984; 44: 1048-53.

15 Haga Y, Sakamoto K, Egami H, Yoshimura R, Mori K, Akagi M. Clinical significance of serum CA 125 values in patients with cancers of the digestive system. Am J Med Sci 1986; 292: 30-4.

16 Braganza JM, Hunt LP, Warwick F. Relationship between pancreatic exocrine function and ductal morphology in chronic pancreatitis. Gastroenterology 1982; 82: 1341-7.

17 Haga Y, Sakamoto K, Egami H, Yoshimura R, Akagi M. Evaluation of serum CA 125 values in healthy individuals and pregnant women. Am J Med Sci 1986; 292: $25-9$.

18 Schmiegel WH, Kreiker C, Eberl W, et al. Monoclonal antibody defines CA $19-9$ in pancreatic juices and sera. Gut 1985; 26: 456-60.

19 Jalanko H, Kuusela P, Roberts P, Sipponen P, Haglund $\mathrm{C}$, Makela $\mathrm{O}$, Comparison of a new tumor marker, CA $19-9,{ }^{\text {TM }}$ with $\alpha$-fetoprotein and carcinoembryonic antigen in patients with upper gastrointestinal diseases. $J$ Clin Pathol 1984; 37: 218-22.

20 Sears HF, Herlyn M, Del Villano B, Steplewski Z, Koprowski H. Monoclonal antibody detection of a circulating tumor-associated antigen. II. A longitudinal evaluation of patients with colorectal cancer. J Clin Immunol 1982; 2: 141-9.

21 Kuusela P, Jalanko H, Roberts $\mathrm{P}$, et al. Comparison of CA 19-9 and carcinoembryonic antigen (CEA) levels in the serum of patients with colorectal diseases. $\mathrm{Br} J$ Cancer 1984; 49: 135-9. 